\title{
Do maser stars trace a rotating Galactic bar?
}

\author{
H. J. Habing \\ Leiden Observatory, the Netherlands \\ email: habing@strw.leidenuniv.nl
}

\begin{abstract}
I have analyzed the radial velocities in two large samples of maser stars that cover the Galactic plane from $l=+40 \mathrm{deg}$ to $l=-40 \mathrm{deg}$ through the Galactic Centre. I compare a diagram of longitude versus radial velocity as observed with a diagram obtained from the calculation of orbits in a simple, but realistic potential containing a weak, rotating bar. Almost all stars belong to the Galactic disk but there is a small sample with high velocities close to the Galactic Centre and in the so-called forbidden quadrants. These velocities cannot be explained by a combination of pure Galactic rotation and a modest dispersion in velocity. An acceptable explanation is one in which the stars of this small sample move up and down a rotating Galactic bar.
\end{abstract}

\section{The problem}

Maser stars are all late AGB-stars at the centre of a sphere of matter continuously ejected by the star. The formation of dust in the envelope makes the stars very faint at wavelengths below $1 \mu \mathrm{m}$ and strong in the infrared. In the extreme cases the peak of the SED can be at 20 to $30 \mu \mathrm{m}$. Stellar maser emission is seen in the $1612 \mathrm{MHz}$ line of $\mathrm{OH}$, in several lines of $\mathrm{SiO}$ and in a $22 \mathrm{GHz}$ line of $\mathrm{H}_{2} \mathrm{O}$. The line emission makes it possible to measure very precisely the radial velocity of each star. The $\mathrm{OH}$ and $\mathrm{SiO}$ maser emission is stable in time, but stellar $\mathrm{H}_{2} \mathrm{O}$-masers vary strongly and irregularly; they may remain undetectable during long periods and then suddenly flare. For that reason $\mathrm{H}_{2} \mathrm{O}$-maser stars will not be discussed here. Stars with maser emission at $1612 \mathrm{MHz}$ will be called $\mathrm{OH} / \mathrm{IR}$ stars. Infrared emission provides the non-thermal excitation of the molecules and appears to be well-understood. Recent spectroscopic studies of $\mathrm{OH} / \mathrm{IR}$ stars at red wavelengths have shown the presence of s-type elements and thus the occurrence of Hot Bottom Burning (Garcia-Hernandez et al., 2006).

Over the last 25 years several radio astronomical surveys have been made for maser stars in the Milky Way in the Northern and the Southern skies. The results have provided me with a data base that contains almost $800 \mathrm{OH} / \mathrm{IR}$ stars detected in a $6^{\circ}$-wide strip along the Galactic equator over the longitude range from $-45^{\circ}$ to $+40^{\circ}$ and a velocity range from -300 to $+300 \mathrm{~km} \mathrm{~s}^{-1}$. At all longitudes there is a strong concentration to the Galactic plane. The longitude distribution shows a clear concentration around the Galactic Centre (GC) proving that our sensitivity is good enough to reach beyond the GC. From earlier surveys we know that the number of detections drops considerably in the anti-centre direction; maser stars are confined to the inner Galaxy. Stars with infrared excess do in general not show this confinement and the scarcity of maser stars in the anti-centre may be due to a lower metallicity the outer Galaxy.

In Figure $1 \mathrm{I}$ show two $l V$-diagrams of radial velocity as a function of longitudesee the legend. The $x$-axis and the pair of inclined lines bracket the bulk of the data; these curves represent the maximum rotational velocity at positive longitudes and the minimum at negative longitudes, if the gas had a constant circular velocity of $200 \mathrm{~km} \mathrm{~s}^{-1}$. 

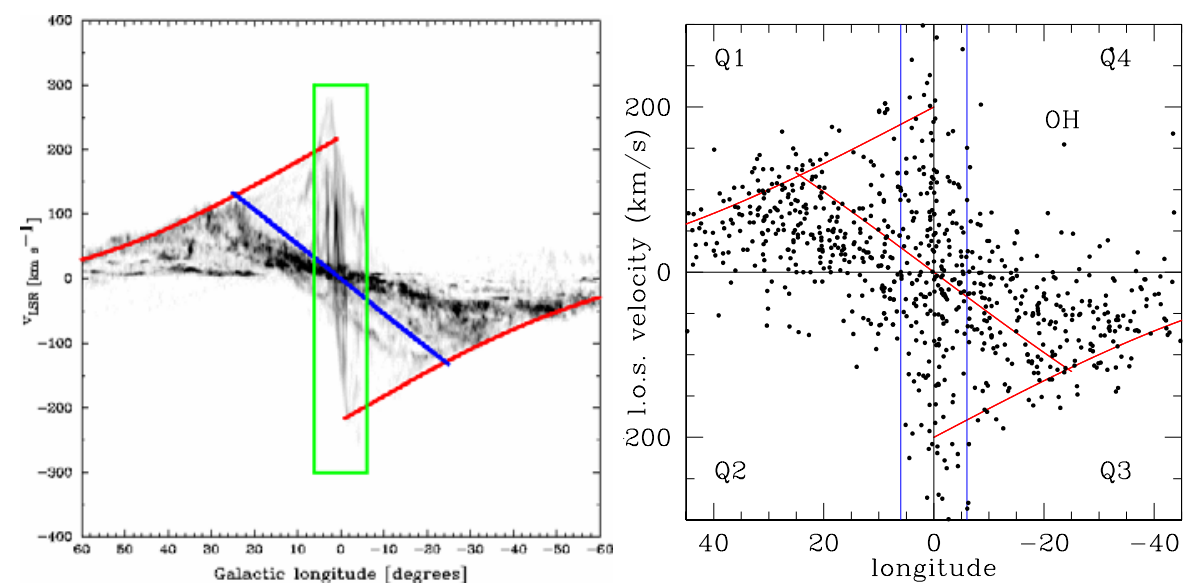

Figure 1. The left diagram shows the intensity of the interstellar CO-line in the Galactic plane as a function of longitude and latitude. For the meaning of the lines see the text.The diagram on the right shows the distribution in longitude and velocity of $\mathrm{OH}$ maser stars. The inclined curves and the transversal and vertical lines have the same meaning as in the diagram on the left.

The transversal line through the centre indicates the upper limit if there were no COemitting gas inside $3 \mathrm{kpc}$ from the Centre and the box marks the innermost part of the diagram, i.e. the area between $-6^{\circ}$ and $+6^{\circ}$. Comparing the two diagrams in Fig. 1 we find the following:

- Almost all CO-emission is confined by the inclined curves and the transversal line,

- A significant number of maser stars cross limits set by Galactic rotation- see the number of stars outside the limits observed by the CO-gas,

- Within $6^{\circ}$ from the GC one finds little gas at "forbidden" velocities, i.e. rectangles defined by: $\left(V \leqslant 0^{\circ}, l \geqslant 0^{\circ}\right)$ and $\left(V \geqslant 0^{\circ}, l \leqslant 0^{\circ}\right)$ but many stars.

The question that we want to answer is: what kinds of stellar orbits will explain the distribution of the stars in the right hand diagram of Fig.1?

\section{Building a model}

The starting point for our study was the significant number of stars with high and forbidden velocities in the area limited by the box in the left diagram of Fig. 1. Could these velocities be explained by stars falling into the GC from the molecular ring at $3 \mathrm{kpc}$ from the GC? Such falling in produces velocities high enough, but the $l V$-diagram is wrong. The next question is: what high and forbidden velocities would they have if these stars were falling in inside a rotating bar? To explore this suggestion we selected some areas in the $l V$-diagrams and counted stars in each area. We then used a simple potential for a $2 \mathrm{D}$ galaxy with a rotating Galactic bar, we calculated orbits for different initial conditions and derived the time spent in each area. By making linear combinations of different orbits we then attempted to reproduce the observed numbers of stars in each area.

The potential we used has been given by Binney and Tremaine (1987). It has the following form:

$$
\Phi=\frac{1}{2} V_{0}^{2} \ln \left\{r_{0}^{2}+x^{2}+(1+\varepsilon) y^{2}\right\}
$$

where $x$ is measured along the bar and $y$ perpendicular to the bar; $\varepsilon$ measures the strength 


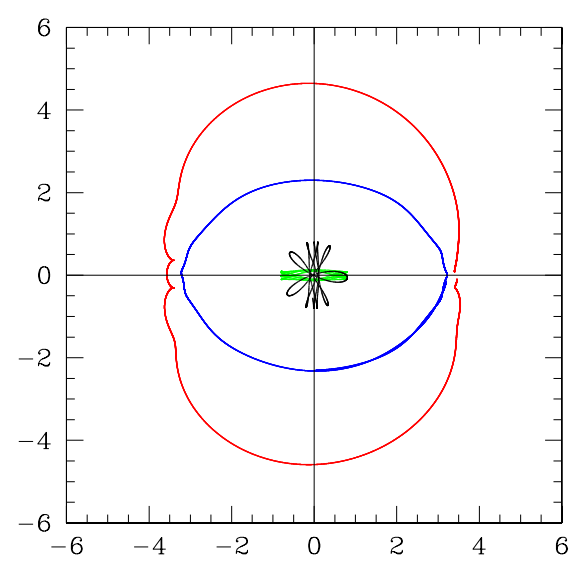

Figure 2. This figure shows two orbits of stars in the Galactic plane. The orbits start at almost the same position on the $x$-axis and with almost identical velocities and yet they cross the $y$-axis at very different locations. See text for explanation.

of the bar. It is assumed that the bar rotates with angular speed $\Omega$. The two equations for the motion are now given by:

$$
\frac{d^{2} x}{d t^{2}}=-\frac{\partial \Phi}{\partial x}+\Omega^{2} x+2 \Omega \frac{d y}{d t}
$$

and by

$$
\frac{d^{2} y}{d t^{2}}=-\frac{\partial \Phi}{\partial y}+\Omega^{2} y-2 \Omega \frac{d x}{d t} .
$$

The free parameters of the model were chosen as: $v_{0}=200 \mathrm{~km} \mathrm{~s}^{-1}, r_{0}=0.15 \mathrm{kpc}$, $\varepsilon=0.11$, and $\Omega=-60 \mathrm{~km} \mathrm{~s}^{-1} \mathrm{kpc}^{-1}$.

\section{Properties of selected orbits}

We started the calculation of the orbits at the $x$-axis assigning the star a velocity in the $y$-direction equal to $f$ times the local circular velocity; $f$ was always chosen in the interval $(0,1)$. Pure circular orbits no longer exist because the potential is not circularly symmetric. However, the deviation from circular symmetry of $\Phi$ is small and quasicircular orbits, or rather orbits limited to a narrow range in distance are always present, inside as well as outside of co-rotation. This is, however, not true around co-rotation at $x=3.33 \mathrm{kpc}$ where $x \Omega=v_{0}$, the co-rotation distance.

Fig. 2 shows two orbits in the plane of the Galaxy that have almost the same initial conditions. They both start at the $x$-axis just inside and just outside the co-rotation distance $(x=3.33 \mathrm{kpc}$ and with the local rotational velocity, i.e. just below and just above $200 \mathrm{~km} \mathrm{~s}^{-1}$. In spite of the very small differences in initial conditions they cross the $y$-axis at distances that differ by almost a factor 2 . There is an explanation for this effect: stars just inside the bar will go faster than the bar and are slowed down by the bar, whereas stars outside co-rotation will go slower than the bar and the bar will thus accelerate the two stars. This speeding-up and slowing-down explains why the two orbits pass the $y$-axis at such different distances. It also implies another property: if the majority of maser stars is on almost circular orbits than we do not expect to find stars in between the two larger orbits in Fig. 2. 

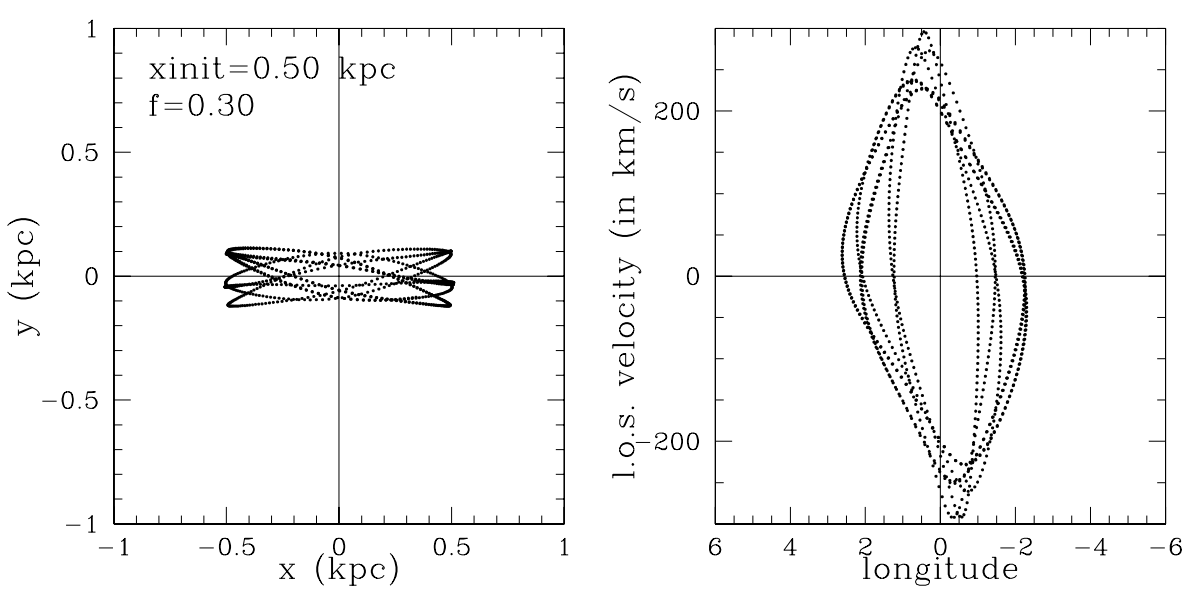

Figure 3. The left diagram shows an orbit in the Galactic plane as described in the text. The right diagram shows the same orbit in lV-space.
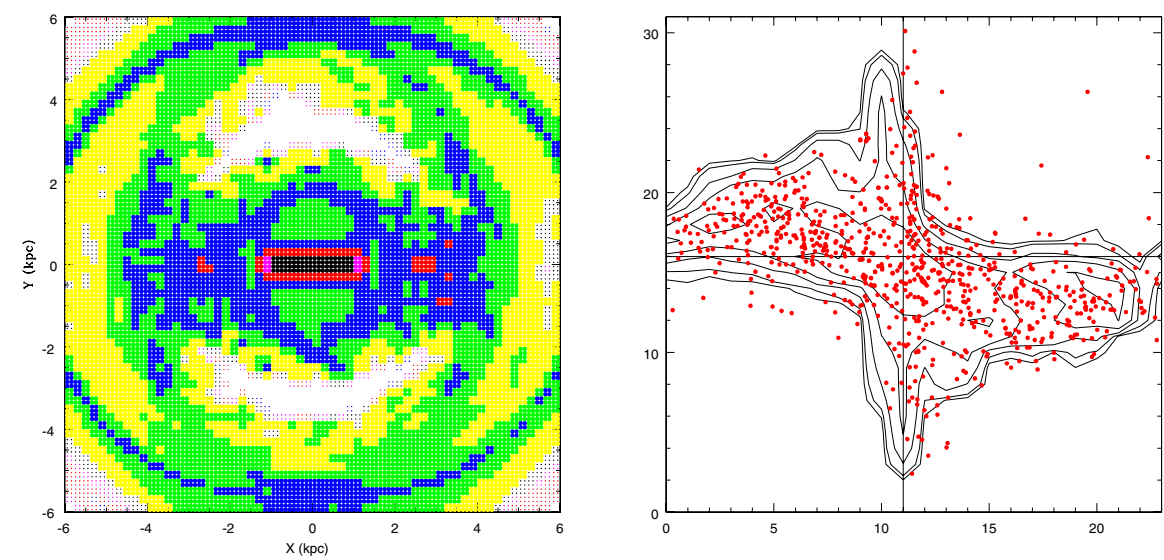

Figure 4. The left diagram shows the distribution of $\mathrm{OH}$ maser stars in the Galactic plane in a frame that rotates with angular velocity $\Omega=-60 \mathrm{~km} \mathrm{~s}^{-1}$. The oval at the centre indicates the highest density; it is the rotating bar. The diagram on the right shows the individual $\mathrm{OH}$ maser stars overlaid over the phase space distribution in longitude and velocity.

Figure 3 shows an interesting type of orbits. They again set forth from the $x$-axis but with a velocity in the $y$-direction that is only 0.3 times the circular velocity. Figure 3 (left diagram) shows this particular orbit in the frame of the (rotating) bar: the star moves up and down along the bar. In doing this it reaches very high and forbidden in the $l V$-diagram: it is the type of orbit that we looked for at the beginning of this article.

\section{Final model}

We calculated numerically many orbits and found that a set of initial conditions, i.e. of initial values $(x, \dot{x}=0, y, \dot{y})$ can be obtained that predict rather well the numbers in the selected areas. This set specifies the distribution of Galactic orbits of the maser stars. As expected, most stars are on almost disk-like orbits but there is a significant number of stars on bar-like orbits such as in Fig. 3 . 
The distribution of initial conditions equals the so-called phase space distribution. Knowing the orbits one can calculate the distribution of the maser stars in the inner Milky Way Galaxy. Figure 4 (left diagram) shows the distribution in the plane of our Galaxy. One easily recognizes the Galactic bar and also the two empty, ear-like areas centred at the corotation point at the $y$-axis. Fig. 4 (right diagram) shows the velocity distribution belonging to the model with the $\mathrm{OH}$ maser velocities superposed.

These results have been published by Habing et al. (2006).

\section{Acknowledgement}

I thank my collaborators Maartje Sevenster, Maria Messineo, Glenn van der Ven and Koen Kuijken

\section{References}

Binney J., \& Tremaine S. 1987, Galactic Dynamics (Princeton University Press)

Garcia-Hernandez, D. A., Garcia-Lario, P., Plez, B., Manchado, A., D’Antona, F., Lub, J., \& Habing, H. J. 2007, A\&A 462, 711

Habing, H., Sevenster, M., Messineo, M., van der Ven, G., \& Kuijken, K. 2006, A\&A, 458, 151 